\title{
Helical Repeat Structure of Apoptosis Inhibitor 5 Reveals Protein-Protein Interaction Modules ${ }^{*[5]}$
}

Received for publication, October 27, 2011, and in revised form, February 9, 2012 Published, JBC Papers in Press, February 12, 2012, DOI 10.1074/jbc.M111.317594

Byeong-Gu Han ${ }^{\ddagger \S 1}$, Kyoung Hoon Kim ${ }^{\Uparrow 1}$, Sang Jae Lee ${ }^{\ddagger 1}$, Kyung-Chae Jeong ${ }^{\ddagger}$, Jea-Won Cho ${ }^{\ddagger}$, Kyung Hee Noh", Tae Woo Kim", Soon-Jong Kim ${ }^{* *}$, Hye-Jin Yoon", Se Won Suh", Sangho Lee ${ }^{\xi}$, and Byung II Lee

From the ${ }^{\ddagger}$ Biomolecular Function Research Branch, Division of Convergence Technology, Research Institute, National Cancer Center, Goyang, Gyeonggi 410-769, Korea, the "Department of Chemistry and Department of Biophysics and Chemical Biology, College of Natural Sciences, Seoul National University, Seoul 151-747, Korea, the "Department of Biomedical Sciences, Graduate School of Medicine, Korea University, Seoul 136-701, Korea, the **Department of Chemistry, Mokpo National University, Chonnam 534-729, Korea, and the ${ }^{\S}$ Department of Biological Sciences, Sungkyunkwan University, Suwon, Gyeonggi 440-746, Korea

Background: Up-regulated in various cancers, API5 prevents apoptosis under growth factor deprivation.

Results: We have determined the crystal structure of API5 with the HEAT and ARM repeat and show that Lys-251 acetylation is important for its function.

Conclusion: API5 likely serves as a scaffold for multiprotein complex with its cellular function regulated by lysine acetylation. Significance: Structural basis of API5 function is important in targeting anti-apoptosis.

Apoptosis inhibitor 5 (API5) is an anti-apoptotic protein that is up-regulated in various cancer cells. Here, we present the crystal structure of human API5. API5 exhibits an elongated all $\alpha$-helical structure. The N-terminal half of API5 is similar to the HEAT repeat and the C-terminal half is similar to the ARM (Armadillo-like) repeat. HEAT and ARM repeats have been implicated in protein-protein interactions, suggesting that the cellular roles of API5 may be to mediate protein-protein interactions. Various components of multiprotein complexes have been identified as API5-interacting protein partners, suggesting that API5 may act as a scaffold for multiprotein complexes. API5 exists as a monomer, and the functionally important heptad leucine repeat does not exhibit the predicted a dimeric leucine zipper. Additionally, Lys-251, which can be acetylated in cells, plays important roles in the inhibition of apoptosis under serum deprivation conditions. The acetylation of this lysine also affects the stability of API5 in cells.

Apoptosis, a programmed cell death process, plays important roles in sculpting the developing organism and in maintaining cell number homeostasis $(1,2)$. It is also critical for effective cancer chemotherapy (3). Two main pathways of apoptosis have been studied extensively. The extrinsic pathway is mediated by interactions between the death receptors and death ligands. This induces the formation of the death-induc-

* This work was supported by the Basic Science Research Program through the National Research Foundation of Korea funded by Ministry of Education, Science, and Technology Grant 2010-0020993 (to B. I. L.).

The atomic coordinates and structure factors (codes 3UOR and 3V6A) have been deposited in the Protein Data Bank, Research Collaboratory for Structural Bioinformatics, Rutgers University, New Brunswick, NJ (http://www.rcsb.org/).

5 This article contains supplemental "Materials and Methods" and Figs. S1-S3.

${ }^{1}$ These authors contributed equally to this work.

${ }^{2}$ To whom correspondence should be addressed: Biomolecular Function Research Branch, Div. of Convergence Technology, Research Inst., National Cancer Ctr., Goyang, Gyeonggi 410-769, Korea. Tel.: 82-31-920-2223; Fax: 82-31-920-2006; E-mail: bilee@ncc.re.kr. ing signaling complex and caspase-8 (4). The intrinsic pathway, which is triggered by radiation, drugs, reactive oxygen species, and radicals, begins with the release of cytochrome $c$ from mitochondria, which ultimately activates caspase- $9(4,5)$. These pathways converge downstream, as both caspase- 8 and caspase- 9 activate caspase-3 $(4,6)$. Activated caspases cleave specific target proteins, such as protein kinases, cytoskeletal proteins, and DNA repair proteins, initiating apoptosis $(6,7)$. The characteristic markers of apoptosis include cell and nuclear shrinkage, DNA cleavage into nucleosomal fragments, chromatin aggregation, and apoptotic body formation (8). Because the precisely regulated events of apoptotic cell death are frequently altered in cancers, proteins in the apoptotic pathway can represent good targets for anti-cancer drugs (9).

Inhibitor of apoptosis (IAP) ${ }^{3}$ family proteins are negative regulators of apoptosis and were characterized originally as physical inhibitors of caspases (10). Eight IAP proteins have been identified, and each of these proteins contains an $\sim 70$ amino acid BIR (baculovirus IAP repeat) domain that mediates protein recognition and interactions (11). Some IAPs include additional domains, such as a RING (really interesting new gene) domain, UBA (ubiquitin-associated domain), or CARD (caspase recruitment domain). Anti-cancer drugs that target IAPs by mimicking the $\mathrm{N}$-terminal IAP binding motif (Ala-ValPro-Ile) of Smac are currently in clinical trials for some solid tumors and lymphomas (12).

API5 (apoptosis inhibitor 5), also known as AAC-11 protein (anti-apoptosis clone 11), FIF (fibroblast growth factor 2-interacting factor), and MIG8 (cell migration-inducing gene $\underline{8}$ ), is a relatively poorly studied apoptosis-inhibiting nuclear protein that does not contain a baculovirus IAP repeat domain. The API5 gene has been found in animals, protists, and plants (13). The expression of API5 prevents apoptosis following growth factor deprivation, and this protein is up-regulated in various

\footnotetext{
${ }^{3}$ The abbreviations used are: IAP, inhibitor of apoptosis; r.m.s.d., root mean square deviation; PDB, Protein Data Bank; EJC, exon junction complex.
} 


\section{Structure of Apoptosis Inhibitor 5}

cancer cells (14-19). Non-small cell lung cancer patients whose cancers express API5 have a poorer prognosis than patients with non-API5-expressing cancers, and overexpression of API5 promotes the invasion and inhibits apoptosis in cervical cancer cells $(15,17,20)$. It has been suggested that API5 suppresses E2F1 transcription factor-induced apoptosis (21). A recent study also suggested that API5 is phosphorylated by PIM2 kinase and inhibits apoptosis in hepatocellular carcinoma cells through NF- $\kappa$ B (nuclear factor $-\kappa B$ ) (22). Various API5 interaction partners have been identified. API5 interacts with high molecular mass forms of fibroblast growth factor 2 (FGF-2), which are involved in cell proliferation and tumorigenesis (16). API5 also binds to and regulates Acinus, a protein involved in chromatin condensation and DNA fragmentation during apoptosis (23). Moreover, the inhibition of API5 increases anticancer drug sensitivity in various cancer cells (23). Recently, the chromatin remodeling enzyme ALC1 (amplified in liver cancer 1) and two DEAD box RNA helicases were identified as binding partners of API5 $(13,24)$. From these data, API5 has been regarded as a putative metastatic oncogene and therefore represents a therapeutic target for cancer treatment (23). The rational design of inhibitors of API5 will be aided by determination of its three-dimensional structure. Additionally, the lack of sequence similarity between API5 and other known proteins has made it difficult to predict its molecular function. Therefore, structural information about API5 will be helpful in elucidating its molecular function.

We determined the crystal structure of human API5 to elucidate the molecular basis of its anti-apoptotic function. API5 contains a multiple helices, forming HEAT and ARM (Armadillo)-like repeats, which are known to function in protein-protein interactions. This study thus reveals that API5 acts as a mediator of protein interactions.

\section{EXPERIMENTAL PROCEDURES}

Protein Expression and Purification-The human API5 gene (full-length (1-504), API5 $\Delta \mathrm{C}(1-454))$ was amplified by PCR and cloned into pET28b $(+)$ (Novagen) using NdeI and NotI restriction sites. This construction adds a 21-residue tag including $\mathrm{His}_{6}$ to the $\mathrm{N}$-terminal of the recombinant protein, facilitating protein purification. The mutants (K251Q, K251R, and $\mathrm{K} 251 \mathrm{~A}$ ) of the API5 gene were prepared by QuikChange site-directed mutagenesis method using the wild type plasmid as the PCR template. The wild type and mutant proteins were overexpressed in Escherichia coli Rosetta2(DE3) cells (Novagen). The cells were grown at $37^{\circ} \mathrm{C}$ in 4 liters of Terrific Broth medium to an $A_{600}$ of 0.7 , and expression of the recombinant protein was induced with $0.5 \mathrm{~mm}$ isopropyl $\beta$-D-thiogalactopyranoside at $37^{\circ} \mathrm{C}$. The cells were grown at $37^{\circ} \mathrm{C}$ for $16 \mathrm{~h}$ after isopropyl $\beta$-D-thiogalactopyranoside induction and were harvested by centrifugation at $3000 \times g$ for $10 \mathrm{~min}$ at $4{ }^{\circ} \mathrm{C}$. The cell pellet was suspended in ice-cold lysis buffer $(25 \mathrm{~mm}$ Tris- $\mathrm{HCl}$, pH 7.4, $138 \mathrm{~mm} \mathrm{NaCl}, 2 \mathrm{~mm} \mathrm{KCl,} \mathrm{10 \%} \mathrm{(v/v)} \mathrm{glycerol,} 1 \mathrm{~mm}$ phenylmethylsulfonyl fluoride, and $0.8 \mu \mathrm{M}$ lysozyme) and homogenized by sonication. The first purification step utilized a $\mathrm{Ni}^{2+}$ nitrilotriacetic acid column (Qiagen) for affinity purification via the $\mathrm{N}$-terminal $\mathrm{His}_{6}$ tag. The eluent was pooled and concentrated. The protein sample was diluted 10-fold with buffer (50 mm Tris-HCl, pH 8.0, $80 \mathrm{~mm} \mathrm{NaCl}$, and $1 \mathrm{~mm}$ DTT). Further purification was conducted using a HiTrap Q ion exchange chromatography column (GE Healthcare) equilibrated with buffer ( $50 \mathrm{~mm}$ Tris- $\mathrm{HCl}, \mathrm{pH} 8.0,80 \mathrm{~mm} \mathrm{NaCl}$, and 1 mM DTT). The protein was eluted using a linear gradient of $0-1.0$ M sodium chloride in the same buffer. The final purification step was gel filtration on a HiLoad 16/60 Superdex 200 prep-grade column (GE Healthcare) equilibrated with buffer containing $20 \mathrm{~mm}$ sodium citrate, $\mathrm{pH} 5.5,200 \mathrm{~mm} \mathrm{NaCl}$, and $1 \mathrm{~mm}$ DTT. The purified API5 protein was concentrated to $\sim 20 \mathrm{mg} / \mathrm{ml}$ using an YM10 membrane (Millipore). Human $\alpha$-thrombin (Enzyme Research Laboratories) or porcine tryp$\sin$ (Promega) was used to remove the fusion tag or to achieve limited proteolysis, respectively.

Crystallization, X-ray Data Collection, and Structure Determination-The best crystals were obtained with a reservoir solution of 10\% PEG 6000 and 100 mm bicine, pH 9.0, using the API $5 \Delta$ C protein. For $\mathrm{x}$-ray diffraction data collection, crystals were transferred to a cryoprotectant solution (10\% PEG 6000, 100 mm Bis-Tris, pH 6.0, and 30\% ethylene glycol) and mounted on nylon loops. Data of API $5 \Delta$ Cs (wild type and K251Q mutant) were collected using an ADSC Quantum CCD detector at the 4A and 6C experimental stations, Pohang Light Source, Korea and an ADSC Quantum 4 CCD detector at the BL-1A experimental station, Photon Factory, Japan, respectively. Intensity data were processed and scaled using the program HKL2000 (25). Selenium sites were located with SOLVE (26) using two MAD data sets. Initial phases were improved using the program RESOLVE (26). Manual model building was performed using the program COOT (27), and the model was refined with the program PHENIX (28) and Refmac (29), including bulk solvent correction. As the test data for the calculation of $R_{\text {free }}, 5 \%$ of the data were randomly set aside (30). The refined models were evaluated using MolProbity (31).

Analytical Ultracentrifugation-Equilibrium sedimentation experiments were performed using a Beckman ProteomeLab XL-A analytical ultracentrifuge in $20 \mathrm{~mm}$ sodium citrate buffer, $\mathrm{pH} 5.5$, containing $200 \mathrm{~mm} \mathrm{NaCl}$, at $20^{\circ} \mathrm{C}$. Absorbances of API5 samples were measured at 235,240 , and $280 \mathrm{~nm}$ using a twosector cell at two different speeds $(12,000$ and 16,000 rpm) and two different concentrations ( 4.90 and $9.80 \mu \mathrm{M})$ with a loading volume of $180 \mu \mathrm{l}$. The calculated partial specific volumes at $20^{\circ} \mathrm{C}$ were 0.7452 and $0.7416 \mathrm{~cm}^{3} / \mathrm{g}$ for API $5 \Delta \mathrm{C}$ and full-length API5, respectively. The buffer density was $1.01424 \mathrm{~g} / \mathrm{cm}^{3}$. For mathematical modeling using non-linear least-squares curve fitting, the fitting function for homogeneous models was used, as described elsewhere (32). The model was selected by examining the weighted sum or square values and weighted root mean square deviation (r.m.s.d.) values.

Cell Viability Test-Jurkat cells were grown in RPMI 1640 media supplemented with $10 \%$ fetal bovine serum (WelGENE). Transient transfection was performed with Lipofectamine LTX and Plus Reagent (Invitrogen) according to the manufacturer's instructions. Briefly, $2.3 \times 10^{5}$ Jurkat cells were seeded in the medium onto a 24-well plate. After $1 \mathrm{~h}$, the cells in each well were transfected with $0.6 \mu \mathrm{g}$ of each DNA construct (fulllength API5 wild type, K251R, K251Q, and K251A, respectively). After $3 \mathrm{~h}$, the serum-free medium was exchanged with 


\section{Structure of Apoptosis Inhibitor 5}

RPMI1640 medium containing 10\% fetal bovine serum to stabilize the transfected cells. After an additional 12-h incubation, the culture medium was exchanged with serum-free RPMI 1640 to perform a starvation test. Following a 48-h incubation, the cell viability in each well was determined using a Presto cell proliferation assay kit (Invitrogen) according to the manufacturer's instructions.

Immunoprecipitation and Western Blotting-To monitor the oligomerization state of API5 in cells, full-length HA-API5 and $3 \times$ FLAG-API5 were co-transfected into HeLa cells and immunoprecipitated. Whole cell lysates prepared in lysis buffer (50 mm Tris- $\mathrm{HCl}, \mathrm{pH} 7.5,150 \mathrm{~mm} \mathrm{NaCl}, 1 \%$ Triton X-100, 0.5\% sodium deoxycholate, and $0.1 \%$ sodium dodecyl sulfate), were used for immunoprecipitation. Each lysate was mixed with a binding buffer (50 mM Tris- $\mathrm{HCl}, \mathrm{pH} 7.5,150 \mathrm{~mm} \mathrm{NaCl}$, and 1 mM EDTA) supplemented with protease inhibitor cocktails (Roche Applied Science). The mixture was incubated overnight at $4{ }^{\circ} \mathrm{C}$ with antibodies (diluted 1:100). A/G-agarose beads were then added, incubated at $4{ }^{\circ} \mathrm{C}$ for $3 \mathrm{~h}$, and washed four times with the binding buffer. The immune complex was released from the beads by boiling in sample buffer and was visualized by Western blotting. Western blotting was carried out using established protocols. The primary antibodies used were anti-HA (Santa Cruz Biotechnology), anti-FLAG (Cell Signaling Technologies), and anti- $\beta$-actin (Abcam).

To test protein stability in cells, full-length wild type API5 or mutants API5 (K251R and K251Q) was transfected into HeLa cells. At $22 \mathrm{~h}$ after transfection, the cells were treated with 10 $\mu \mathrm{g} / \mathrm{ml}$ of cycloheximide for $4 \mathrm{~h}$. Then, the cells were collected, and protein levels were analyzed by Western blotting. For the deacetylase inhibition experiments, suberoylanilide hydroxamic acid was pretreated for $20 \mathrm{~h}$ to the wild type API5-transfected HeLa cells before cycloheximide treatment.

\section{RESULTS}

Structure Determination and Overall Structure-We obtained crystals of full-length and trypsin-treated API5. However, only the crystals of trypsin-treated API5 were suitable for structure determination. From N-terminal amino acid sequencing and MALDI-TOF analysis, we found that the $\mathrm{N}$-terminal fusion tag and $\sim 50$ amino acid residues at the C-terminal of recombinant API5 were removed by trypsin and that the trypsin-treated form of API5 corresponded to residues $1-454$. The crystal structure of API5 $\Delta \mathrm{C}$ was solved by multiwavelength anomalous dispersion and refined to 2.50 and 2.60 $\AA$ resolution for wild type and K251Q (acetylation mimic) mutant structures, respectively. The refined models of wild type and K251Q mutant exhibited working and free $R$-values of 21.6 and $24.2 \%$, and 21.3 and $25.0 \%$, respectively, with good stereochemistry (Table 1). One subunit of API5 $\Delta \mathrm{C}$ was found to be present in the asymmetric unit of the crystal. The structures of wild type and K251Q mutant are highly similar to each other, with an r.m.s.d. of $0.32 \AA$ for $424 \mathrm{C}_{\alpha}$ atom pairs (supplemental Fig. S1).

The API $5 \Delta$ C monomer presented an elongated, all $\alpha$-helical repeat structure, forming a right-handed superhelix with approximate dimensions of $100 \times 35 \times 50 \AA$ (Fig. $1 A$ ). It consisted of $19 \alpha$-helices $(\alpha 1-\alpha 19)$ and two $3_{10}$ helices. Each helix was observed to be paired with its neighboring helix to form an antiparallel helix pair. Residues 277-278, 322-335, 365-366, $430-431$, and 447-454, as well as the four extra N-terminal residues (Gly-Ser-His-Met), artificially added by cloning into pET-28b, were disordered in the crystal. The C-terminal region of API5 (residues 455-504), which contains a nuclear localization signal (Fig. 2) between residues $454-475$ and is predicted to be disordered by IUPRED (33), was readily removed by trypsin. Additionally, an electron density map calculated using low resolution $(\sim 6 \AA)$ x-ray diffraction data from full-length API5 did not show this $\mathrm{C}$-terminal region, supporting its hypothesized flexibility (data not shown). Several positively charged patches were found on the concave surface of the API5 $\Delta \mathrm{C}$ protein, whereas mainly negatively charged residues were positioned on the convex side (Fig. $1 B$ ). The positively or negatively charged patches were well conserved in various species (Fig. $1 C)$. Basic patches were also found near the putative leucine zipper region (heptad leucine repeat). However, a basic DNA binding region typically followed by a leucine zipper of other leucine repeat proteins, was not found to be present in API5 (Fig. 2).

Searches using the DALI server were carried out to identify structurally similar proteins (34). Because the structure of API5 $\Delta \mathrm{C}$ can be divided into two regions, DALI searches were performed separately for the N-terminal half $(\alpha 1-\alpha 11)$ and the C-terminal half $(\alpha 12-\alpha 19)$. The N-terminal half of API5 structurally aligns with the HEAT repeat regions of importin $\beta$ (PDB code 1 O6P, Z-score $=13.1$; r.m.s.d., $4.7 \AA$ for 190 structurally aligned residues), the TOG2 domain of Msps (PDB code 2QK2, Z-score $=12.2$; r.m.s.d. $=4.5 \AA$ for 176 structurally aligned residues), and protein phosphatase $2 \mathrm{~A}$ (PDB code 2C5W, Z-score $=11.8$, r.m.s.d. $=1.8 \AA$ for 87 structurally aligned residues). Although the sequence identities between the structurally aligned regions and API5 were only $10-12 \%$, the overall folds were quite similar. The C-terminal half of API5 is structurally similar to the core region of the U-box-containing ubiquitin ligase E4 protein Ufd2p (PDB code 2QIZ, residues 461747). The Z-score and r.m.s.d. values for the aligned 195 amino acid pairs were 8.3 and $4.4 \AA$, respectively. The amino acid sequences were also quite different between API5 and Ufd2p (lower than 11\% sequence identity), although the three-dimensional structures were quite similar, including the characteristic long helix pair $(\alpha 18-\alpha 19)$. The second most similar protein to the C-terminal half of API5 was p120 catenin (PDB code 3L6Y, $\mathrm{Z}$-score $=7.7$, r.m.s.d. $=3.3 \AA$ for the aligned 152 amino acid pairs). Ufd2p and p120 catenin have been classified as ARMlike repeat proteins. These results indicate that the $\mathrm{N}$-terminal half of API5 adopts the characteristic HEAT repeat structure, which is composed of pairs of antiparallel helices, whereas the C-terminal half of API5 shows an ARM-like repeat structure. Superposition of API5 and the structurally similar protein is shown in Fig. 3. The interhelix turns between antiparallel helices are relatively short (between two and four residues) in the HEAT repeats of API5, resulting in a tight fold of the repeat structure, whereas the turns in the ARM-like repeat regions are longer (6 to 22 residues) (Fig. 2).

The amino acid sequence of API5 contains an LxxLL motif (16). The LxxLL motif forms a short amphipathic $\alpha$-helix and is 


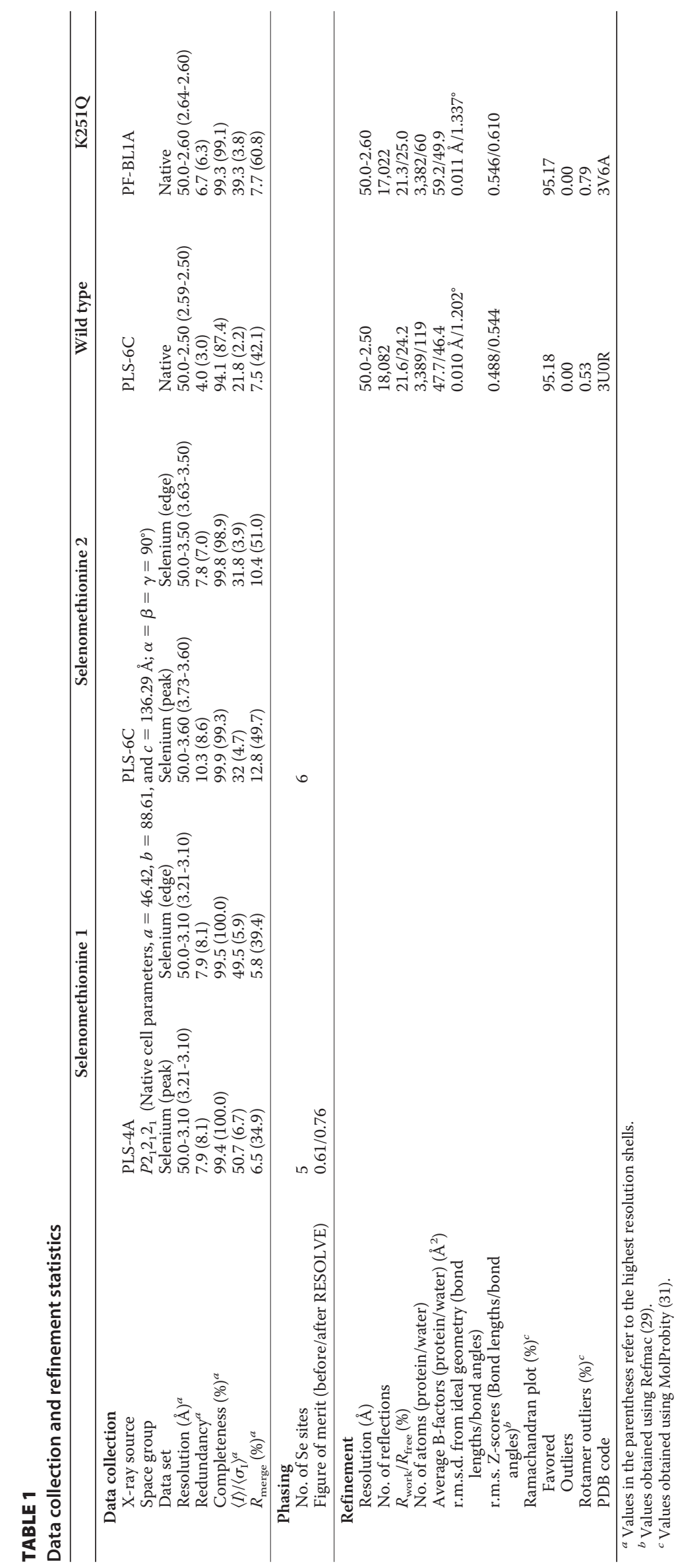


A
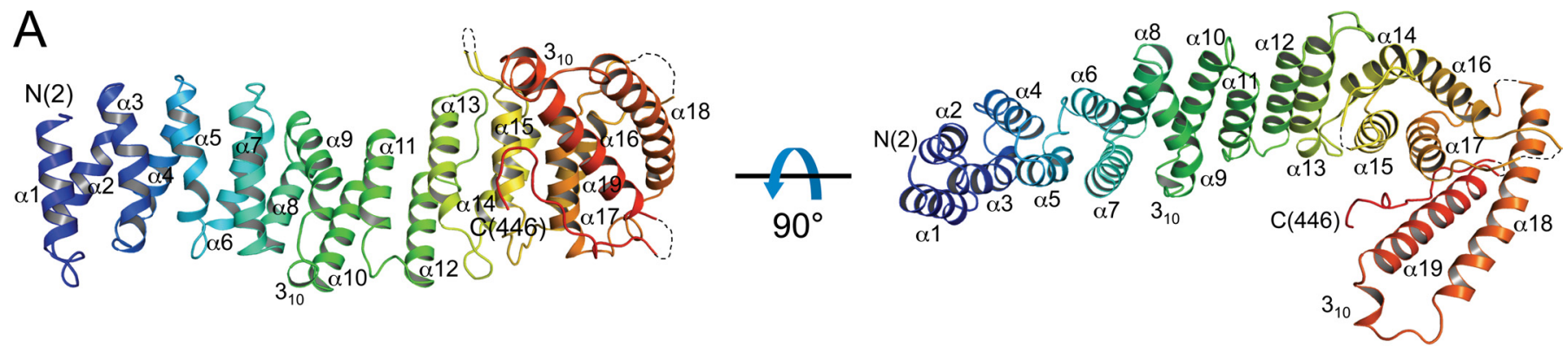

B
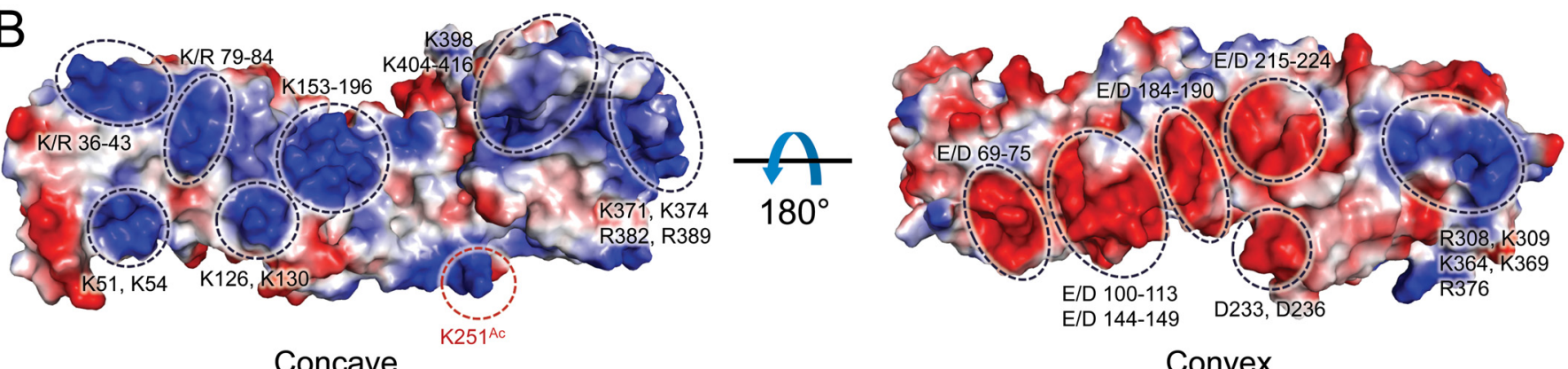

Convex
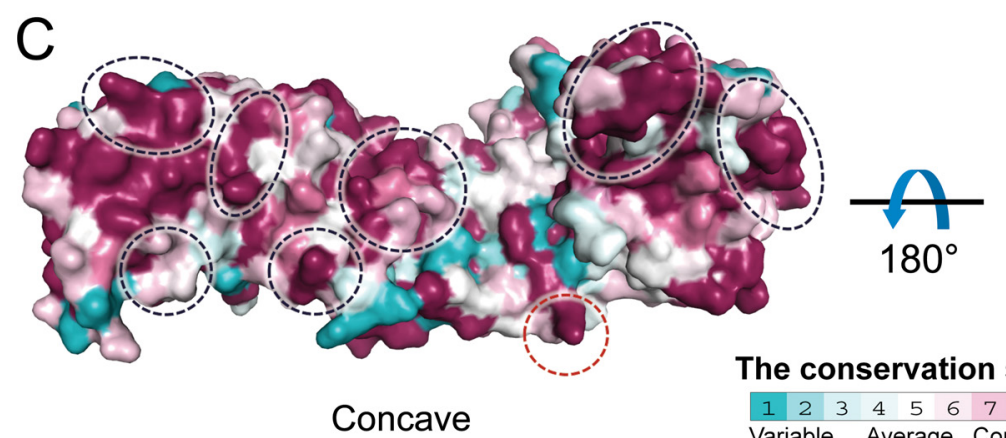

The conservation scale
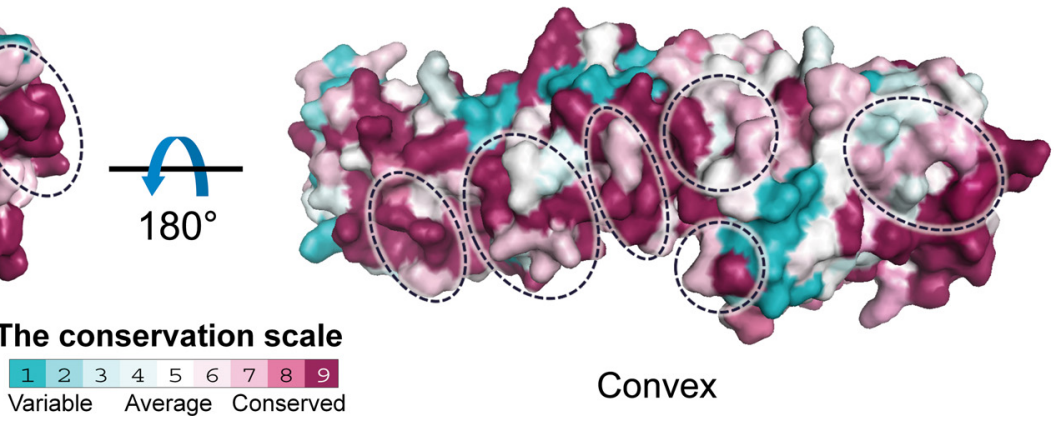

Convex

FIGURE 1. The overall structural representation of API5. $A$, the ribbon diagram of API5 $\Delta$ C reveals its elongated, all $\alpha$-helical structure. $B$, surface charge properties of API5 $\triangle$ C. The concave region of API5 is highly positively charged, whereas the convex region is negatively charged. The acidic and basic residues in the surface are denoted. Lys-251, shown using red dashed circles, is solvent-exposed, allowing access by acetyltransferases or deacetylases. C, surface representation colored by amino acid sequence conservation. Surface conservation was generated using ConSurf (57).

usually found in nuclear receptor-cofactor interaction regions $(35,36)$. The L $x x$ LL motif in API5 is positioned in $\alpha 6$ (Figs. $1 A$, $2,4 A$ ). However, the three leucine residues in this motif are not located on the surface of the protein and likely do not play any role in protein-protein interactions. Instead, because they are located in the interior of the protein, they may contribute to the stability of the HEAT repeat by forming hydrophobic interactions with neighboring $\alpha$-helices. Leu-102 interacts with Ala120 , Ile-124 of $\alpha 7$, and Leu-86 of $\alpha 5$. Leu-105 and Leu-106 interact with Val-117 and Phe-114 of $\alpha 7$, respectively (Fig. 4A). This finding is consistent with a previous report indicating that the replacement of these conserved leucine residues does not abolish FGF-2 binding (16).

Assessment of API5 Oligomerization and Heptad Leucine Repeat Region-The heptad repeat of leucine residues between residues 370 and 391 in API5 has been predicted to be a leucine zipper without a basic DNA-binding region (14). Therefore, it has been suggested that API5 may form a dimer in solution. However, the crystal structure of API $5 \Delta$ C indicates that API5 is monomeric and that the putative leucine zipper $(\alpha 18)$ does not interact with the corresponding helix of another subunit.
Instead, it interacts with $\alpha 19$ of the same subunit (Figs. $1 A$ and $4 B$ ). Two sets of low resolution data were collected from crystals of full-length API5 ( $\sim 6 \AA$ resolution) or API5 $\Delta \mathrm{C}(\sim 4 \AA$ resolution) grown under different crystallization conditions. Protein molecules positioned by the molecular replacement method indicate that both API5 and API5 $\Delta \mathrm{C}$ are monomeric under different crystallization conditions (data not shown).

Because the oligomeric state of API5 in the crystal was found to be different from the predicted dimeric state, the relevant oligomeric state of API5 in solution was further investigated by analytical ultracentrifugation (Fig. $5 A$ ). The estimated molecular mass $(54,949 \mathrm{Da}( \pm 234))$ agreed with the calculated molecular mass for an API5 $\Delta \mathrm{C}$ monomer, including the fusion tag $(53,521 \mathrm{Da})$. The full-length API5 protein was also found to be monomeric, indicating minimal effects of the C-terminal truncation on the oligomeric structure of API5. The estimated molecular mass of full-length API5 $(60,129 \mathrm{Da}( \pm 1,164))$ agreed with the calculated molecular mass for the full-length API5 monomer including the fusion tag $(58,934 \mathrm{Da})$.

Additionally, we transiently expressed HA-tagged and $3 \times$ FLAG-tagged full-length API5 in HeLa cells and performed 
$\alpha 1$

Human

Human

Mouse

Chicken

Salmon

Drosophila $\alpha 2$

$\alpha 3$

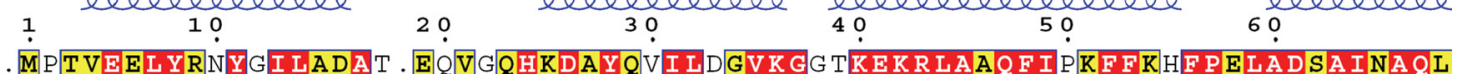

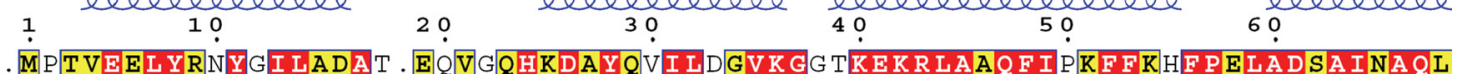

$\alpha 4$

lelelele MPTVEELYRNYGI LADAT. EQVGQHKDAY QVI LDGVKGGTKEKRLAAQFIPKFFKHF PELADSAINA $Q L$ MPTVEELYRNYGI LADAT. ETAGQHKDAYQVILDGVKGGAKEKRLAAQF I PKF FKHF PELADSA INA QL

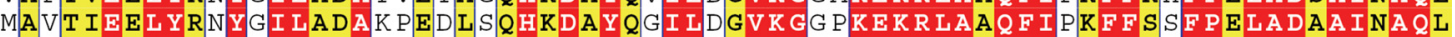
. MDN IERLYK CYEI LSEAG . DKISEHVDEYKEI LKAVKGT SKEKR LASQ FI GNFFKHF PDLA DTA IDA QF

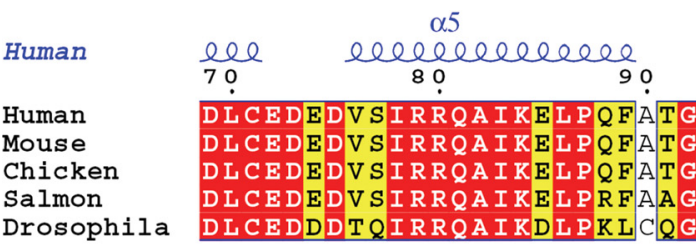

Human

Human

Mouse

Chicken

Salmon

Drosophila

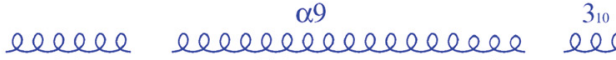
140 150 $\alpha 6$

$\alpha 6$
elevelevele
100 $\frac{\alpha 7}{\alpha 8}$

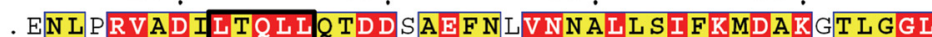
. . EN L P R A I L T Q L L T D D A EF N LVNNALLSIFKMDAKGTLGG L

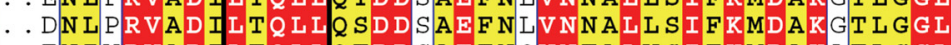
EN I VR VAD IL TQ L L T T D S A F N QVNTALV S F K MDAK A T L G G L NADA T IRVGDTLAOLLI LD D TELQQVNNSLLAIIKLDTKSSIAG L

\section{LxxLL}

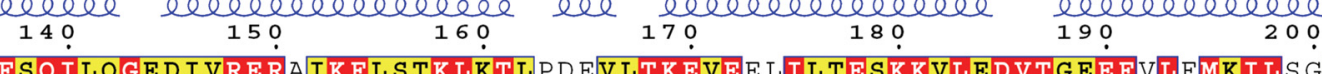

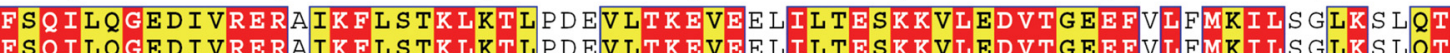

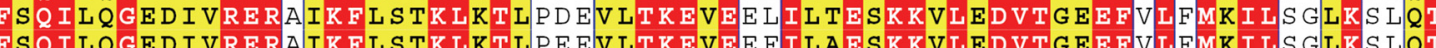
TESKRLEDVTGEEFVLFMKILSGLKS

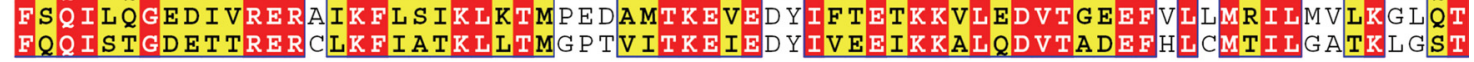

Human

Human

Mouse

Chicken

Salmon

Drosophila $\alpha 12$

210
210

230 lebelee

$\alpha 13$ $\alpha 14$

${ }_{260}$

VSGRQQLVELVAEQADLEQT . . . FNPSDPDCVDRLLQCTRQAVPLFSKNVHSTRFVTYFCEQVLPN . LG VSGRQQLVELVAEQADLEQA. . . FSPSDPDCVDRLLQCTRQAVPLFSKNVHSTRFVTYFCEQVLPN. . LS VSGRQQLVELVAEQADLEQT. . F F P SDPDCVDRLLQCTROAVPLFSKNVHSTKFVTYFCEHVLPN. LS MSGRQQLVELVVEQAFIEQA. . . LNPADPDTVDRLLQCTRQALPLFSKNVHSTRFVTYFCDHVLPN. . LS

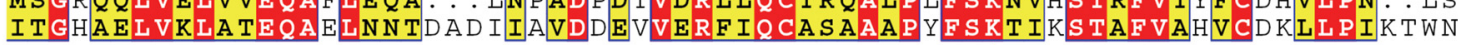

$\triangle$

$\alpha 15$

Human

Human

Mouse

Chicken

Salmon

Drosophila

$\alpha 16$

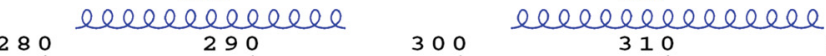

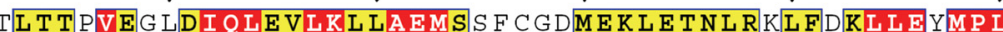
TL T TPVEGLDI QLEVLKLLAEMS S F CGDMEKLETNLRKLFDKLLEYMPLPPE. EAENGENAGNEEPKLOE ALT TPVEG LD I Q LEVLKLLAEMS S F CGDMEKLESNLKKLFDKLLEYMPLPPE. EAENGENAGGEEPKLO SL T S PVAELDI Q LEVLKLLAEMS PF CGDMEKVETNLTMLFEKLLELMP L P PEA EGENGENTL SDEPKLO MI A TAVS QDQI QLRLLKVFAEMITNTDKLDNASER INAVYNVL LEYMPL PKL. . . . SDEDLGDTPPSFQF

$\alpha 17$

Human

Human

Mouse

Chicken

Salmon

Drosophila $\alpha 18$

3

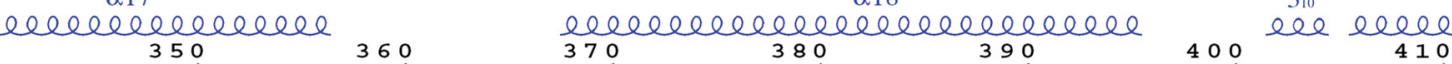
YYVTCLY SFUL SYVECLLY SFHQLGRK LPDFLTAKLNAEKLKD FK IRLQYFARGLQVY IRQLR LAL Q GK T GEALKTEENKI SYVECLIF SFHQL GK L P D F I D K I NA ERLKDFKIRLQYFARGLQVYIRQLRVALQGKT GDALKTE ENRI SHAECLLYALFTLGKNHPNSLSFVEDAEKLKD FRAR LQYLARGTOGY IKKLEESLKGKT GEELKTEENQL

$\alpha 19$

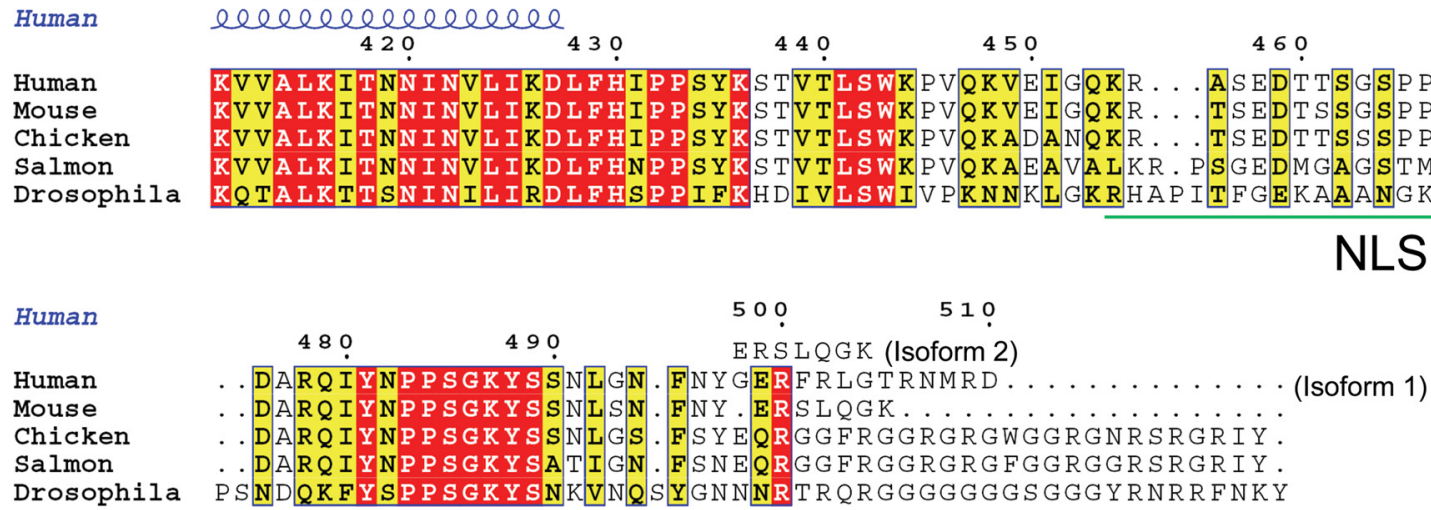




\section{Structure of Apoptosis Inhibitor 5}
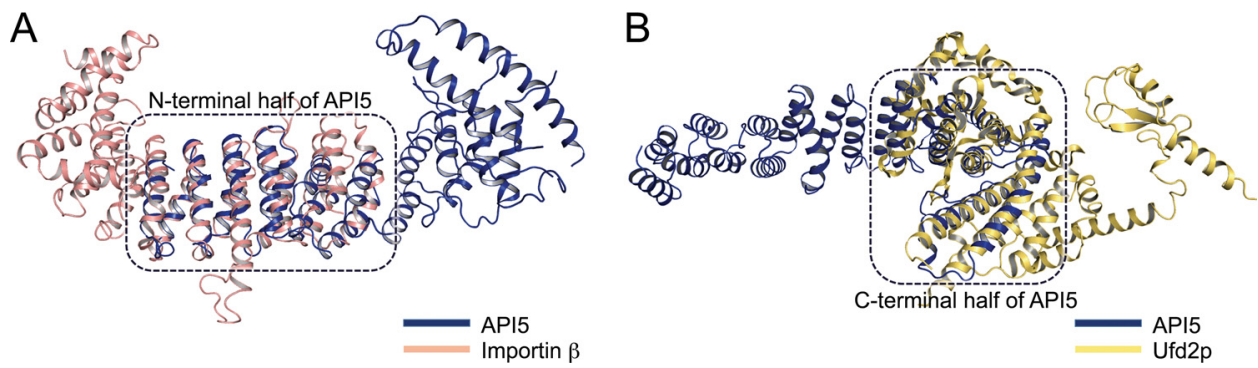

FIGURE 3. The structural similarity between API5 and HEAT and ARM motif-containing proteins. $A$, the N-terminal half of API5 is structurally similar to importin $\beta$ (salmon). B, the C-terminal-half of API5 is structurally similar to the core domain of Ufd $2 \mathrm{p}$ (yellow). Superimposed regions are indicated by dashed boxes.
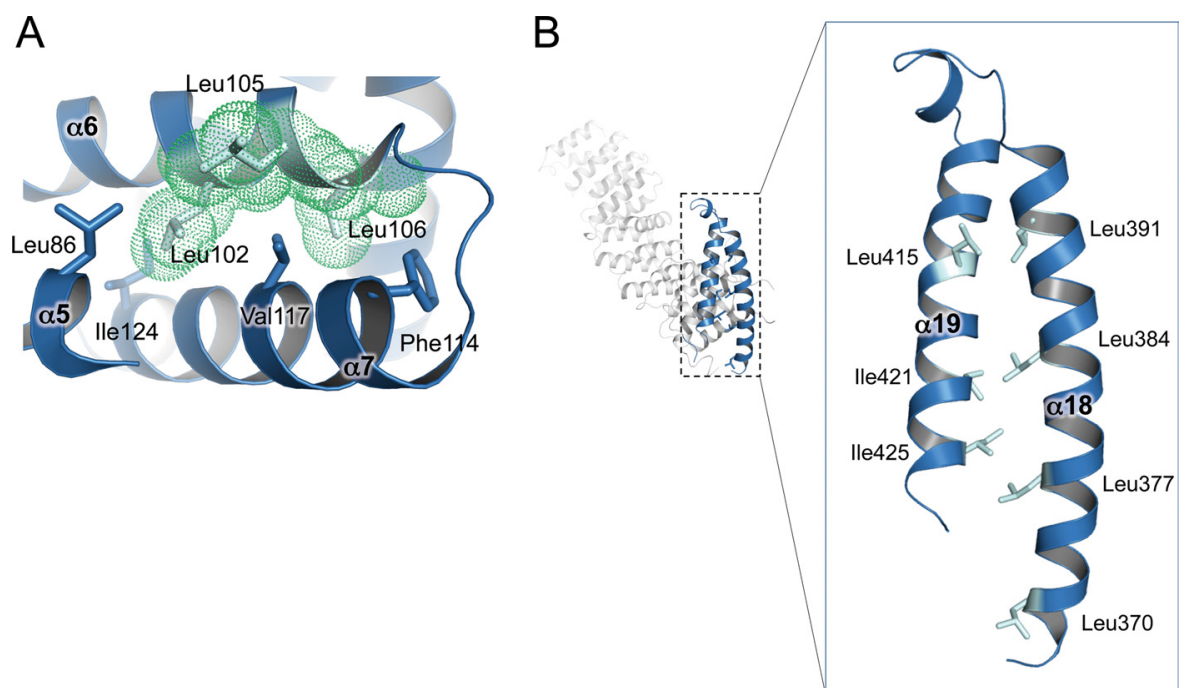

FIGURE 4. Detailed motif/domain structure of API5. $A$, LxxLL motif and interacting residues. The leucine residues interact with hydrophobic residues on the adjacent helices and are involved in stabilization of the repeated structure. $B$, heptad leucine repeat region. $\alpha 18$ is covered by $\alpha 19$ from the same subunit and does not form a typical leucine zipper. The leucine residues of the heptad repeat and hydrophobic residues on $\alpha 19$ are close to each other (shown in a stick representation).

immunoprecipitation experiments (Fig. $5 B$ ). No interaction was found between HA-tagged API5 and $3 \times$ FLAG-tagged API5. This result shows that API5 does not homodimerize in cells. However, we cannot rule out the possibility that API5 heterodimerize with other leucine zipper-containing proteins under specific cellular conditions. Taken together, we unexpectedly discovered that API5 is monomeric and that its putative leucine zipper helix $(\alpha 18)$ does not participate in dimerization. Three of the four leucine residues in the heptad repeat (Leu-377, Leu-384, and Leu-391) exhibit hydrophobic interactions with hydrophobic residues in $\alpha 19$; Leu-377 and Leu-384 interact with Ile-425 and Ile-421, respectively, and Leu-391 is close to Leu-415 (Fig. 4B). Leu-370 does not interact with other residues.

Lysine Acetylation and Its Effect on Cancer Cells-A global mass analysis showed that Lys-251 of API5 is acetylated in cells (37). Amino acid sequence alignment showed that this lysine residue is strictly conserved across a number of species (Fig. 2). Lys-251 is positioned in a loop between $\alpha 13$ and $\alpha 14$, which is extended from the protein (Fig. 1B). This find- ing suggests that acetyltransferases or deacetylases can easily access this residue.

API5 plays important roles in the inhibition of cell death under serum deprivation conditions $(14,15)$. Therefore, we tested whether lysine acetylation affected cell viability $48 \mathrm{~h}$ after the transient transfection under serum deprivation conditions (Fig. 6A). We made an acetylation-deficient, a constitutive acetylation mimic, and an uncharged mutant (K251R, K251Q, and K251A, respectively) $(37,38)$. Wild type API5 inhibited cell death by serum starvation compared with untreated or control vector-transfected cells. The K251R mutant exhibited even higher cell viability. However, the K251Q mutant did not inhibit apoptosis efficiently compared with the wild type API5 and the K251R mutant. The K251A mutant did not have antiapoptotic function. This result means that the inhibition of apoptosis by API 5 can be negatively regulated by Lys- 251 acetylation. We tried to find the structural basis for anti-apoptotic function by lysine acetylation from the structure of K251Q mutant. However, no significant structural differences were found (supplemental Fig. S1).

FIGURE 2. Amino acid sequence alignment of API5 homologs from different species. The LxxLL motif is marked with a black box. The conserved leucine residues of the heptad leucine repeat are marked by blue circles, and the acetylation site Lys- 251 is marked by a blue triangle. The LxxLL motif and nuclear localization signal (NLS) sequence are marked. The amino acid sequence for isoform 2 of human API5, which was used in this study, is also denoted above the sequence. The figure was prepared using ESPript. 

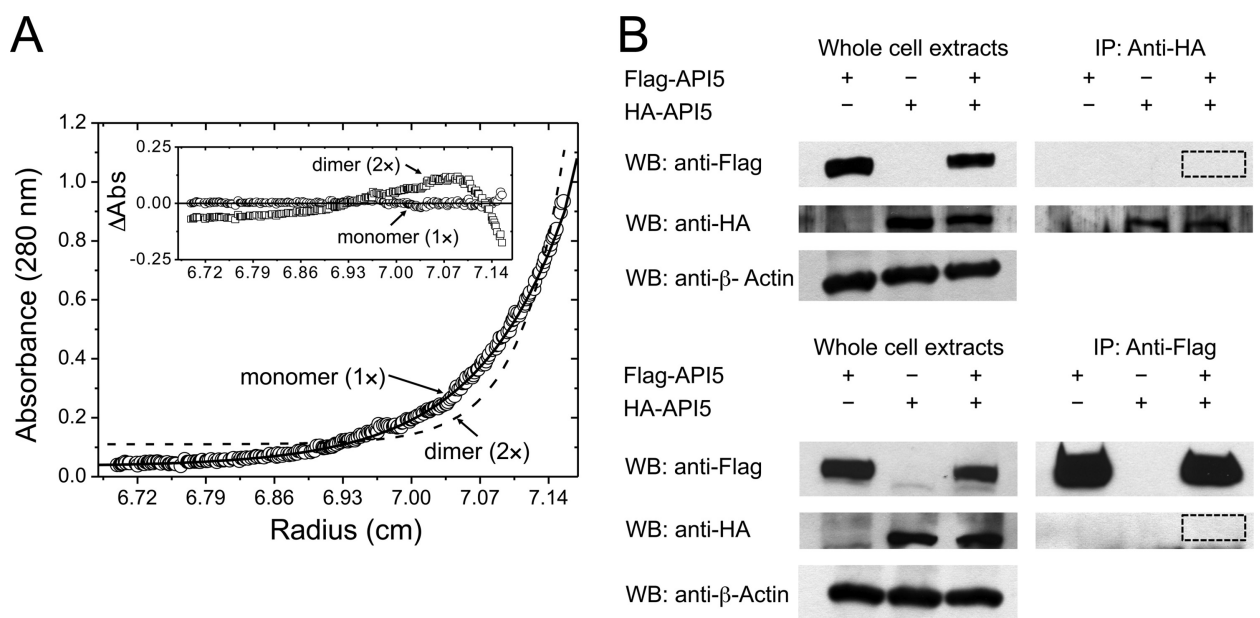

FIGURE 5. Oligomerization of API5. $A$, analytical ultracentrifugation analysis. Distributions of the residuals according to both a monomer (1 $\times$, circle) and a dimer ( $2 \times$, square) models. The random distributions of residuals for the monomer $(1 \times)$ model indicate that API5 $\Delta C$ exists as a homogeneous monomer in solution. $B$, immunoprecipitation (IP) and Western blot (WB) analysis with anti-FLAG and anti-HA API5. No interaction was found between FLAG-API5 and HA-API5, ruling out the possibility of API5 homodimerization.

A

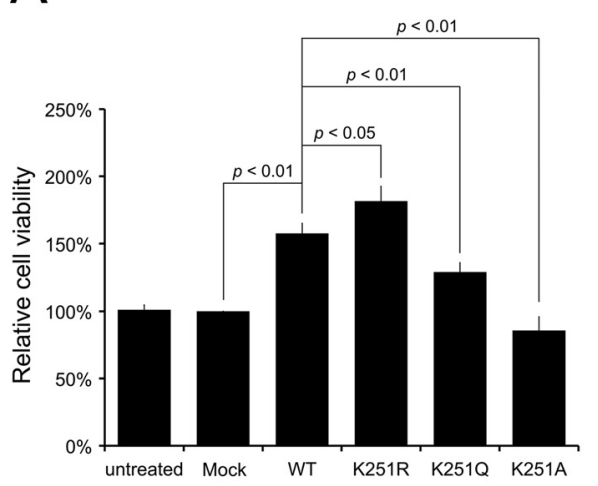

B

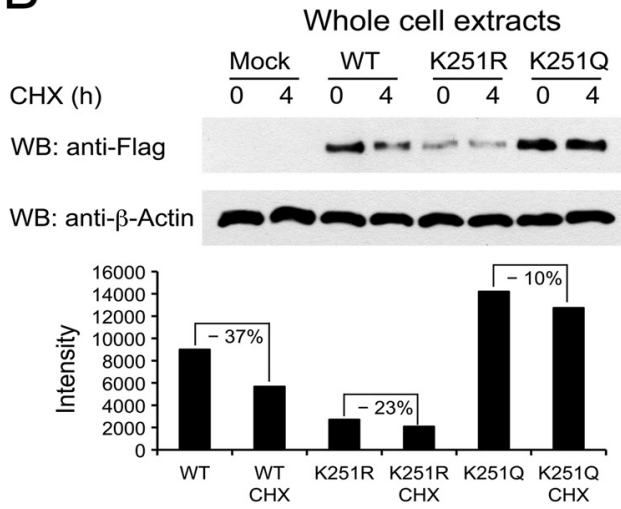

C

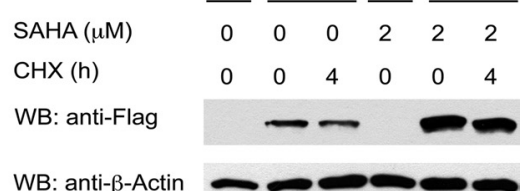

WB: anti- $\beta$-Actin

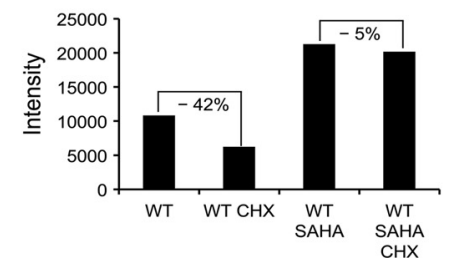

FIGURE 6. Effects of API5 acetylation at Lys-251. Wild type API5, an acetylation-deficient mutant (K251R), a constitutive acetylation mimic mutant (K251Q), and an uncharged mutant (K251 A) were transiently expressed. A, effects on cell viability under conditions of serum deprivation. The K251Q and K251 A mutants do not inhibit apoptosis efficiently. B, effects of Lys-251 acetylation on protein stability. Cycloheximide (CHX) treatment was used to inhibit protein synthesis, and Western blot $(W B)$ /normalized densitiometry analysis was performed to monitor protein stability in cells. $C$, effects on protein stability when deacetylase inhibitor, suberoylanilide hydroxamic acid $(S A H A)$, was co-treated with cycloheximide to wild type API5-transfected cells.

We also tested whether lysine acetylation affected API5 protein stability. After $22 \mathrm{~h}$ of transfection, protein synthesis was stopped by treatment with cycloheximide, and the protein level was monitored by Western blot analysis after $4 \mathrm{~h}$ of treatment. The K251Q mutant was more stable than the wild type API5 and the K251R mutant (Fig. 6B). Similar results were also found when deacetylase inhibitor suberoylanilide hydroxamic acid was treated (Fig. 6C). Therefore, we conclude that the regulation of API5 function by lysine acetylation may occur via effects on protein stability. However, we could not detect any ubiquitination of API5 at Lys-251, suggesting the proteasome-independent degradation pathway (data not shown).

The cellular localization of API5 also was monitored to determine whether lysine acetylation affected the nuclear transport of API5. All of the wild type and mutants API5 (K251R and K251Q) were observed in the cell nuclei, implying that lysine acetylation did not affect cellular localization (supplemental Fig. S2). The analysis of the API5 and FGF-2 interaction detected by in vitro immunoprecipitation method using the purified recombinant API5 and FGF-2 did not show any significant FGF-2 binding differences between wild type and mutant API5s (supplemental Fig. S3).

\section{DISCUSSION}

In this study, we determined the crystal structure of human API5 and found that API5 contains protein-protein interaction modules, such as HEAT and ARM repeats (Fig. 1A). Different types of helix repeat, including HEAT, ARM, ankyrin, tetratrico peptide, and leucine-rich variant repeats, have been found in many proteins and have diverse functional roles in mediating protein-protein interactions (39). The elongated structure of API5 is well suited for interactions with multiple binding partners, similar to the roles of other repeat proteins (40).

The structures of various HEAT and ARM repeat-containing proteins in complexes with other proteins have been elucidated. Ran-importin $\beta$, protein phosphatase 2 holoenzyme, and the Cand1-Cul1-Roc1 complex are good examples of HEAT repeat complex structures (importin $\beta$, the A subunit of protein phosphatase 2 holoenzyme, and Cand 1 are HEAT repeat-containing proteins) (41-43). Several crystal structures of the 


\section{Structure of Apoptosis Inhibitor 5}

ARM repeat-containing protein (e.g. $\beta$-catenin) in complex with the interacting proteins have been determined (44-47). In many curved HEAT repeat proteins, protein-protein interactions are mediated by the concave surface of the protein, regardless of the surface charge distribution, and the proteinprotein interaction surface involving the ARM repeat proteins usually span the entire range of the repeat. The charged concave or convex side of the API5 is well conserved across a number of species. This suggests that binding partners can bind to these regions.

It has been reported that high molecular mass FGF-2 interacts with API5 in the nucleus, and immunoprecipitation results suggest that two separate regions corresponding to three helices $(\alpha 6$ and $\alpha 15-\alpha 16)$ of API 5 are important for FGF- 2 binding (16). The $\alpha 6$ is exposed to the convex surface of API5, and $\alpha 15-\alpha 16$ are exposed on both the concave and convex surfaces. Analyses of surface properties indicated that the convex side of API5 is highly negatively charged, whereas FGF-2 is highly positively charged (Fig. 1B). Although hydrophobic interactions may also be important for binding, we predict that the positively charged high molecular mass FGF-2 likely binds to the negatively charged convex surface of API5, as determined from previous immunoprecipitation results and surface electrostatic potential (16).

It has been reported that API5 binds to Acinus and protects it from cleavage by caspase- 3 , resulting in an inhibition of apoptosis (23). Acinus has been identified as a component of an ASAP (apoptosis and splicing-associated protein) complex and an exon junction complex (EJC) $(48,49)$. API5 and Acinus interact with each other through the heptad leucine repeat region of API5, which seems to be important for API 5 function and may therefore represent a potential therapeutic target for anti-cancer drugs (23). Mutation of leucine residues (Leu384 and Leu-391) in the heptad leucine repeat region abrogates the anti-apoptotic effects of API5 $(14,23)$. Because Leu-384 and Leu-391 contribute to hydrophobic interactions with other hydrophobic residues in $\alpha 19$, mutation of these residues might cause a distortion of the local structure of API5, thus affecting protein-protein interactions, rather than inhibiting the dimerization. Because API5 is monomeric and the heptad leucine repeat of API5 is structurally distinct from that of other leucine zippers, API5 provides a unique opportunity for anticancer drug discovery through targeting of this region. However, further studies and target validation will be required to develop API5-targeted inhibitors.

It is known that $\mathrm{N} \epsilon$-acetylation at lysine residues affects DNA binding, protein-protein interaction, cellular localization, ubiquitination, phosphorylation, and protein stability (50). A global analysis of lysine acetylation using high resolution mass spectrometry showed that Lys-251 of API5 is acetylated (37). Our data clearly showed that lysine acetylation impacts cell survival under serum deprivation conditions, suggesting an inhibition of function. Therefore, we conclude that API5 function is negatively regulated by acetylation at Lys-251. Inhibition of function via acetylation has also been found in HSP90 and 14-3-3 proteins $(37,38)$. We determined that lysine acetylation affects API5 protein stability, which has been found for p53 as well (51). However, we could not detect ubiquitination of API5, and a detailed mechanism for degradation of API 5 has not yet been established. We predict that API5 may be readily acetylated by an acetyltransferase(s) after protein synthesis and that API5 exists in an inactive and stable state in cells until certain deacetylases can activate API5 through deacetylation. However, activated API5 appears to be unstable and easily degraded in cells. Although the acetyltrasferases or deacetylases that act on API5 have not yet been identified, these enzymes are likely to be important regulatory factors for the function of API5. Additionally, we predict that certain histone deacetylase inhibitors may also be effective in regulating API5 function. We could not found any significant differences in API5-FGF-2 interaction and cellular localization from the lysine acetylation mimic/deficient mutants. However, it is still possible that lysine acetylation of API5 will affect protein-protein interactions between API5 and other API5 interacting partners to induce anti-apoptosis function. We can easily predict that lysine acetylation can inhibit the binding of API5 to its binding partners for its proper function. However, it is also quite likely that lysine acetylated API5 can be recognized by new binding partners, such as bromodomain containing proteins, resulting in a negative regulation of apoptosis (52). Hence, further investigations are needed to elucidate the detailed mechanism for functional regulation by lysine acetylation.

A recent report suggested that the rice API5 homolog interacts with two DEAD-box RNA helicases AIP1/2 (ㅅI5-interacting protein $1 / 2$ ), thereby regulating programmed cell death in the tapetum during the development of male gametophytes (13). The human homolog of rice AIP1/2 is the ATP-dependent RNA helicases UAP56. This protein is involved in pre-mRNA splicing and mRNA nuclear export (53). Interestingly, hUAP56 is a component of large protein complexes, such as the EJC or TREX (Transcription-export) complex $(54,55)$. Particularly, Acinus, another API5 binding protein, is also a component of EJC complex, implying the functional link between API5 and EJC complex. Another study showed that API5 interacts with the chromatin-remodeling enzyme ALC1 (also known as CHD1L), which is a member of the SNF2 family containing a DEAH box helicase domain (24). ALC1 plays important roles in promoting cell proliferation and inhibits apoptosis in hepatocellular carcinoma (56). ALC1 also has been shown to interact with various DNA repair proteins such as DNA-PKCs, Ku, PARP1 (Poly [ADP-ribose] polymerase 1), XRCC (X-ray repair cross-complementing protein), and APLF (Aprataxin PNK-like factor) as well as API5 (24), implying that API5 may also mediate protein-protein interactions in DNA damage response protein complexes. Taken together, the interactions between API5 and EJC component proteins and chromatin-remodeling enzyme such as ALC1 suggest the possible roles of API5 at a transcriptional level.

In conclusion, the protein structure obtained in this study reveals that API5 possesses protein-protein interaction modules that likely mediate interactions with partners such as high molecular mass FGF-2, Acinus, AIP1/2, and ALC1. These interacting proteins are usually components of multiprotein complexes. Therefore, API5 may serve as a scaffold for multiprotein complexes, and the identification of interacting protein partners will help to illuminate the functional roles of API5. 
Furthermore, since we show that the API5 function is regulated by lysine acetylation, identifying the lysine acetyltransferases and deacetylases that act on API5 should also yield a better understanding of the mechanisms that regulate API5 function.

Acknowledgments-We thank the staff at the Pohang Light Source (beamlines $4 A$ and $6 C$ ) and the Photon Factory (BL-1A) for assistance with synchrotron data collection.

\section{REFERENCES}

1. Meier, P., Finch, A., and Evan, G. (2000) Apoptosis in development. Nature $407,796-801$

2. Danial, N. N., and Korsmeyer, S. J. (2004) Cell death: Critical control points. Cell 116, 205-219

3. Igney, F. H., and Krammer, P. H. (2002) Death and anti-death: Tumor resistance to apoptosis. Nat. Rev. Cancer 2, 277-288

4. Yang, S. Y., Sales, K. M., Fuller, B., Seifalian, A. M., and Winslet, M. C. (2009) Apoptosis and colorectal cancer: Implications for therapy. Trends Mol. Med. 15, 225-233

5. Altieri, D. C. (2010) Survivin and IAP proteins in cell death mechanisms. Biochem. J. 430, 199-205

6. Ghobrial, I. M., Witzig, T. E., and Adjei, A. A. (2005) Targeting apoptosis pathways in cancer therapy. CA Cancer J. Clin. 55, 178-194.

7. Alnemri, E. S., Livingston, D. J., Nicholson, D. W., Salvesen, G., Thornberry, N. A., Wong, W. W., and Yuan, J. (1996) Human ICE/CED-3 protease nomenclature. Cell 87, 171

8. Wyllie, A. H., Kerr, J. F., and Currie, A. R. (1980) Cell death: The significance of apoptosis. Int. Rev. Cytol. 68, 251-306

9. Kasibhatla, S., and Tseng, B. (2003) Why target apoptosis in cancer treatment? Mol. Cancer Ther. 2, 573-580

10. Salvesen, G. S., and Duckett, C. S. (2002) IAP proteins: Blocking the road to death's door. Nat. Rev. Mol. Cell Biol. 3, 401-410

11. Srinivasula, S. M., and Ashwell, J. D. (2008) IAPs: What's in a name? Mol. Cell 30, 123-135

12. Gyrd-Hansen, M., and Meier, P. (2010) IAPs: From caspase inhibitors to modulators of NF- $\kappa$ B, inflammation, and cancer. Nat. Rev. Cancer 10, 561-574

13. Li, X., Gao, X., Wei, Y., Deng, L., Ouyang, Y., Chen, G., Li, X., Zhang, Q., and Wu, C. (2011) Rice APOPTOSIS INHIBITOR5 coupled with two DEAD-box adenosine 5' -triphosphate-dependent RNA helicases regulates tapetum degeneration. Plant Cell 23, 1416-1434.

14. Tewari, M., Yu, M., Ross, B., Dean, C., Giordano, A., and Rubin, R. (1997) AAC-11, a novel cDNA that inhibits apoptosis after growth factor withdrawal. Cancer Res. 57, 4063-4069

15. Kim, J. W., Cho, H. S., Kim, J. H., Hur, S. Y., Kim, T. E., Lee, J. M., Kim, I. K., and Namkoong, S. E. (2000) AAC-11 overexpression induces invasion and protects cervical cancer cells from apoptosis. Lab. Invest. 80, 587-594.

16. Van den Berghe, L., Laurell, H., Huez, I., Zanibellato, C., Prats, H., and Bugler, B. (2000) FIF (fibroblast growth factor-2 (FGF-2)-interacting-factor), a nuclear putatively antiapoptotic factor, interacts specifically with FGF-2. Mol. Endocrinol. 14, 1709-1724.

17. Sasaki, H., Moriyama, S., Yukiue, H., Kobayashi, Y., Nakashima, Y., Kaji, M., Fukai, I., Kiriyama, M., Yamakawa, Y., and Fujii, Y. (2001) Expression of the antiapoptosis gene, AAC-11, as a prognosis marker in non-small cell lung cancer. Lung Cancer 34, 53-57

18. Clegg, N., Ferguson, C., True, L. D., Arnold, H., Moorman, A., Quinn, J. E., Vessella, R. L., and Nelson, P. S. (2003) Molecular characterization of prostatic small-cell neuroendocrine carcinoma. Prostate 55, 55-64

19. Krejci, P., Pejchalova, K., Rosenbloom, B. E., Rosenfelt, F. P., Tran, E. L., Laurell, H., and Wilcox, W. R. (2007) The antiapoptotic protein Api5 and its partner, high molecular weight FGF2, are up-regulated in B cell chronic lymphoid leukemia. J. Leukoc. Biol. 82, 1363-1364

20. Wang, Z., Liu, H., Liu, B., Ma, W., Xue, X., Chen, J., and Zhou, Q. (2010) Gene expression levels of CSNK1A1 and AAC-11, but not NME1, in tumor tissues as prognostic factors in NSCLC patients. Med. Sci. Monit. 16,
CR357-364

21. Morris, E. J., Michaud, W. A., Ji, J. Y., Moon, N. S., Rocco, J. W., and Dyson, N. J. (2006) Functional identification of Api5 as a suppressor of E2F-dependent apoptosis in vivo. PLoS Genet. 2, e196

22. Ren, K., Zhang, W., Shi, Y., and Gong, J. (2010) Pim-2 activates API-5 to inhibit the apoptosis of hepatocellular carcinoma cells through NF- $\kappa \mathrm{B}$ pathway. Pathol. Oncol. Res. 16, 229-237

23. Rigou, P., Piddubnyak, V., Faye, A., Rain, J. C., Michel, L., Calvo, F., and Poyet, J. L. (2009) The antiapoptotic protein AAC-11 interacts with and regulates Acinus-mediated DNA fragmentation. EMBO J. 28, $1576-1588$

24. Ahel, D., Horejsí, Z., Wiechens, N., Polo, S. E., Garcia-Wilson, E., Ahel, I., Flynn, H., Skehel, M., West, S. C., Jackson, S. P., Owen-Hughes, T., and Boulton, S. J. (2009) Poly(ADP-ribose)-dependent regulation of DNA repair by the chromatin remodeling enzyme ALC1. Science 325, 1240-1243

25. Otwinowski, Z., and Minor, W. (1997) Processing of $x$-ray diffraction data collected in oscillation mode. Methods in Enzymology 276, 307-326

26. Terwilliger, T. C., and Berendzen, J. (1999) Automated MAD and MIR structure solution. Acta Crystallogr. D Biol. Crystallogr. 55, 849-861

27. Emsley, P., and Cowtan, K. (2004) Coot: Model-building tools for molecular graphics. Acta Crystallogr. D Biol. Crystallogr. 60, 2126-2132

28. Adams, P. D., Afonine, P. V., Bunkóczi, G., Chen, V. B., Davis, I. W., Echols, N., Headd, J. J., Hung, L. W., Kapral, G. J., Grosse-Kunstleve, R. W., McCoy, A. J., Moriarty, N. W., Oeffner, R., Read, R. J., Richardson, D. C., Richardson, J. S., Terwilliger, T. C., and Zwart, P. H. (2010) PHENIX: A comprehensive Python-based system for macromolecular structure solution. Acta Crystallogr. D Biol. Crystallogr. 66, 213-221

29. Murshudov, G. N., Vagin, A. A., and Dodson, E. J. (1997) Refinement of macromolecular structures by the maximum likelihood method. Acta Crystallogr. D Biol. Crystallogr. 53, 240-255

30. Brünger, A. T. (1992) Free $R$ value: A novel statistical quantity for assessing the accuracy of crystal structures. Nature $355,472-475$

31. Chen, V. B., Arendall, W. B., 3rd, Headd, J. J., Keedy, D. A., Immormino, R. M., Kapral, G. J., Murray, L. W., Richardson, J. S., and Richardson, D. C. (2010) MolProbity: All-atom structure validation for macromolecular crystallography. Acta Crystallogr. D Biol. Crystallogr. 66, 12-21

32. Jung, W. S., Hong, C. K., Lee, S., Kim, C. S., Kim, S. J., Kim, S. I., and Rhee, S. (2007) Structural and functional insights into intramolecular fructosyl transfer by inulin fructotransferase. J. Biol. Chem. 282, 8414-8423

33. Dosztányi, Z., Csizmok, V., Tompa, P., and Simon, I. (2005) IUPred: Web server for the prediction of intrinsically unstructured regions of proteins based on estimated energy content. Bioinformatics 21, 3433-3434

34. Holm, L., and Rosenström, P. (2010) Dali server: Conservation mapping in 3D. Nucleic Acids Res. 38, W545-549

35. Heery, D. M., Kalkhoven, E., Hoare, S., and Parker, M. G. (1997) A signature motif in transcriptional co-activators mediates binding to nuclear receptors. Nature $\mathbf{3 8 7}, \mathbf{7 3 3 - 7 3 6}$

36. Plevin, M. J., Mills, M. M., and Ikura, M. (2005) The LxxLL motif: A multifunctional binding sequence in transcriptional regulation. Trends Biochem. Sci. 30, 66-69

37. Choudhary, C., Kumar, C., Gnad, F., Nielsen, M. L., Rehman, M., Walther, T. C., Olsen, J. V., and Mann, M. (2009) Lysine acetylation targets protein complexes and co-regulates major cellular functions. Science 325, $834-840$

38. Scroggins, B. T., Robzyk, K., Wang, D., Marcu, M. G., Tsutsumi, S., Beebe, K., Cotter, R. J., Felts, S., Toft, D., Karnitz, L., Rosen, N., and Neckers, L. (2007) An acetylation site in the middle domain of Hsp90 regulates chaperone function. Mol. Cell 25, 151-159

39. Groves, M. R., and Barford, D. (1999) Topological characteristics of helical repeat proteins. Curr. Opin. Struct. Biol. 9, 383-389

40. Tu, D., Li, W., Ye, Y., and Brunger, A. T. (2007) Structure and function of the yeast U-box-containing ubiquitin ligase Ufd2p. Proc. Natl. Acad. Sci. U.S.A. 104, $15599-15606$

41. Vetter, I. R., Arndt, A., Kutay, U., Görlich, D., and Wittinghofer, A. (1999) Structural view of the Ran-Importin $\beta$ interaction at 2.3 A resolution. Cell 97, 635-646

42. Cho, U. S., and Xu, W. (2007) Crystal structure of a protein phosphatase 2A heterotrimeric holoenzyme. Nature 445, 53-57 


\section{Structure of Apoptosis Inhibitor 5}

43. Goldenberg, S. J., Cascio, T. C., Shumway, S. D., Garbutt, K. C., Liu, J., Xiong, Y., and Zheng, N. (2004) Structure of the Cand1-Cul1-Roc1 complex reveals regulatory mechanisms for the assembly of the multisubunit cullin-dependent ubiquitin ligases. Cell 119, 517-528

44. Choi, H. J., Gross, J. C., Pokutta, S., and Weis, W. I. (2009) Interactions of plakoglobin and $\beta$-catenin with desmosomal cadherins: Basis of selective exclusion of $\alpha$ - and $\beta$-catenin from desmosomes. J. Biol. Chem. 284, $31776-31788$

45. Sampietro, J., Dahlberg, C. L., Cho, U. S., Hinds, T. R., Kimelman, D., and $\mathrm{Xu}, \mathrm{W}$. (2006) Crystal structure of a $\beta$-catenin-BCL9-Tcf4 complex. Mol. Cell 24, 293-300

46. Xing, Y., Clements, W. K., Kimelman, D., and Xu, W. (2003) Crystal structure of a $\beta$-catenin-axin complex suggests a mechanism for the $\beta$-catenin destruction complex. Genes Dev. 17, 2753-2764

47. Huber, A. H., and Weis, W. I. (2001) The structure of the $\beta$-catenin-Ecadherin complex and the molecular basis of diverse ligand recognition by $\beta$-catenin. Cell 105, 391-402

48. Schwerk, C., Prasad, J., Degenhardt, K., Erdjument-Bromage, H., White, E., Tempst, P., Kidd, V. J., Manley, J. L., Lahti, J. M., and Reinberg, D. (2003) ASAP, a novel protein complex involved in RNA processing and apoptosis. Mol. Cell Biol. 23, 2981-2990

49. Tange, T. Ø., Shibuya, T., Jurica, M. S., and Moore, M. J. (2005) Biochemical analysis of the EJC reveals two new factors and a stable tetrameric protein core. RNA 11, 1869-1883

50. Yang, X. J. (2004) Lysine acetylation and the bromodomain: A new partnership for signaling. Bioessays 26, 1076-1087

51. Yang, X. J., and Seto, E. (2008) Lysine acetylation: Codified cross-talk with other post-translational modifications. Mol. Cell 31, 449-461

52. Zeng, L., and Zhou, M. M. (2002) Bromodomain: An acetyllysine binding domain. FEBS Lett. 513, 124-128

53. Shen, H. (2009) UAP56, a key player with surprisingly diverse roles in pre-mRNA splicing and nuclear export. BMB Rep 42, 185-188

54. Strässer, K., Masuda, S., Mason, P., Pfannstiel, J., Oppizzi, M., RodriguezNavarro, S., Rondón, A. G., Aguilera, A., Struhl, K., Reed, R., and Hurt, E. (2002) TREX is a conserved complex coupling transcription with messenger RNA export. Nature 417, 304-308

55. Le Hir, H., and Andersen, G. R. (2008) Structural insights into the exon junction complex. Curr. Opin. Struct. Biol. 18, 112-119

56. Ma, N. F., Hu, L., Fung, J. M., Xie, D., Zheng, B. J., Chen, L., Tang, D. J., Fu, L., Wu, Z., Chen, M., Fang, Y., and Guan, X. Y. (2008) Isolation and characterization of a novel oncogene, amplified in liver cancer 1, within a commonly amplified region at 1q21 in hepatocellular carcinoma. Hepatology 47, 503-510

57. Ashkenazy, H., Erez, E., Martz, E., Pupko, T., and Ben-Tal, N. (2010) ConSurf 2010: Calculating evolutionary conservation in sequence and structure of proteins and nucleic acids. Nucleic Acids Res. 38, W529-533 